www.nature.com/jhg

\title{
Hypomethylation of the KCNQ1OT1 imprinting center of chromosome 11 associated to Sotos-like features
}

\author{
Journal of Human Genetics (2012) 57, 153-156; doi:10.1038/jhg.2011.145; published online 5 January 2012
}

In a screening for imprinting alterations in 268 patients with idiopathic intellectual disability, we have found a loss of methylation in KCNQ1OT1 (MIM 604115) in one patient with clinical characteristics resembling Sotos syndrome. Sotos syndrome (MIM 117550) is mainly characterised by overgrowth (tall stature and macrocephaly), learning difficulties and facial gestalt (frontal bossing, high hairline, downslanting palpebral fissures, prognathism and pointed chin). ${ }^{1}$ Hypomethylation of KCNQ1OT1, on the other hand, is the most common cause of BeckwithWiedemann syndrome (BWS, MIM 130650), but it previously was reported in two patients diagnosed with Sotos syndrome $^{2,3}$ (Table 1). NSD1 gene deletions or point mutations account for $70-90 \%$ of cases of Sotos syndrome, whereas the disease-causing mechanism of other cases remains unknown. ${ }^{4}$

The patient is the third child of non-consanguineous healthy parents. He was born in the $36^{\text {th }}$ week of gestation with a birth weight of $2940 \mathrm{~g}\left(75-50^{\text {th }}\right.$ percentile) and a length of $51 \mathrm{~cm}\left(>90^{\text {th }}\right.$ percentile), neonatal occipitofrontal circumference was unregistered. He could not walk until 16 months and now, at 12 years of age, he has poor coordination. He also had language delay and did not speak until 3 years.

Clinical examination at 12 years of age noted frontal bossing, sparse hair in frontoparietal area, macrocephaly and dolichocephaly. He also showed foetal finger pads, although not large hands (Figure 1). At that age, his height was $160 \mathrm{~cm}$ ( $>97^{\text {th }}$ percentile). He is moderately mentally retarded and attends a special school. He presents sociability problems, ocular twitch and sometimes he talks alone. The absence of the typical facial gestalt (downslanting palpebral fissures and pointed chin), neonatal hypotonia, large hands or cardiac anomalies do not allow a clinical diagnosis of Sotos syndrome, in spite of the clear similarities.

Standard GTG-banding karyotype from peripheral blood cells was reported normal $(46, \mathrm{XY})$. Subtelomeric rearrangements and classical microdeletion/microduplication syndromes (including Sotos syndrome) were discarded by multiplex ligation-dependent probe amplification (MLPA SALSA P036D, P064 and P245; MRC-Holland, Nederlands). Screening for dosage alterations was performed by a full coverage human-genome oligo-CGH-array from Agilent Technologies (44K oligo array G4410B; Palo Alto, CA, USA) with no relevant findings. All the laboratory analyses so far performed (routine biochemical and genetics analyses) were normal.

Therefore, the patient was included in a series of patients with idiopathic intellectual disability associated to congenital anomalies, in which we performed a screening for DNA methylation alterations in four differentially methylated regions (DMRs): KCNQ1OT1 (11p15), MEG3 (14q32), H19 (11p15) and SNRPN (15q12) based on a methylation test described previously in Martínez et al. ${ }^{5}$ The screening was performed by a multiplexed semi-quantitative PCR amplification, with and without digestion by methylationsensitive enzyme HpaII, using fluorescently labelled primers and capillary electrophoresis. Data analysis was performed in Excel (manuscript in preparation).

The present case showed a hypomethylation of KCNQ1OT1 (Figure 2a). His parents were also studied and no alteration of the methylation or the gene dosage in these regions was found. The results of the patient were confirmed by MLPA (SALSA MS030), with a demethylation of the KvDMR1 region of the KCNQ1OT1 gene of the $80 \%$ (Figures
$2 \mathrm{~b}$ and c). Also, it should be noted that, to date, no false positives have been detected for this diagnostic procedure in 254 non-affected individuals. Three microsatellite markers in this region were studied in the patient and his parents (D11S1984, D11S2362 and D11S1999), showing no evidence of a paternal uniparental disomy (data not shown).

Only two patients with a hypomethylation of KCNQ1OT1 associated to Sotos syndrome have been so far reported in a clinicallyselected series, one of them due to a paternal isodysomy. ${ }^{1,2}$ That study was based on the clinical similarities between BWS and Sotos syndromes, because both conditions share common clinical features such as macrosomia. We have found a third patient with this association in a screening of 268 patients with intellectual disability associated to highly variable clinical symptoms. ${ }^{6,7}$ This finding further confirms the association between Sotos-like symptoms and epigenetic alterations in $11 \mathrm{p} 15$. Most importantly, as far as we know this is the first study that finds such association avoiding any clinical selection bias, which highlights its biological significance.

The molecular basis for this association is unknown. It might well be speculated that some unknown gene, implicated in maintaining the methylation of DMRs, can also cause Sotos or Sotos-like syndrome. Up to date, only three genes are known to cause imprinting disorders by trans-acting loss of imprinting: $Z F P 57,{ }^{8} N A L P 2^{9}$ and NALP7. ${ }^{10}$ Mutation screening by direct sequencing of these genes in the patient did not allow us to find any pathogenic mutation. On the other hand, as the three presently known patients are sporadic, it cannot be rejected to be consequence of a new kind of epimutation without genetic cause. In any case, the methylation test of 
Table 1 Comparison of phenotype of our patient and the patients described by Baujat et al., ${ }^{2,3}$ with the main features of the Sotos Syndrome and the BWS

\begin{tabular}{|c|c|c|c|c|c|}
\hline \multicolumn{4}{|c|}{ Cases described by Baujat et al.2,3 } & \multirow[b]{2}{*}{ Present case } & \multirow[b]{2}{*}{$\begin{array}{c}\text { Beckwith-Wiedemann } \\
\text { syndrome }\end{array}$} \\
\hline & $\begin{array}{c}\text { Sotos } \\
\text { syndrome }\end{array}$ & $F D$ & $R D$ & & \\
\hline \multicolumn{6}{|l|}{ Clinical features } \\
\hline Prenatal overgrowth & + & + & + & + & + \\
\hline Gestational age & & 34 weeks & 40 weeks & 36 weeks & \\
\hline Birth weight (g) & & 2140 (75-50"th percentile) & 3380 (75-50th percentile) & 2940 (75-50th percentile) & \\
\hline Birth length $(\mathrm{cm})$ & & 44 (50-25 $5^{\text {th }}$ percentile) & 49 (50 $0^{\text {th }}$ percentile) & 51 (>90 $0^{\text {th }}$ percentile) & \\
\hline Postnatal Overgrowth & + & + & + & + & + \\
\hline Age & & 11 years & 10 years & 12 years & \\
\hline Height (cm) & & 156 (97 $^{\text {th }}$ percentile) & $146.5{\text { ( } 97^{\text {th }}}_{\text {percentile) }}$ & $160\left(>97^{\text {th }}\right.$ percentile) & \\
\hline \multicolumn{6}{|l|}{ Sotos clinical features } \\
\hline Macrocephaly & + & + & + & + & \\
\hline Dolichocephaly & + & & + & + & \\
\hline Frontal bossing & + & + & + & + & \\
\hline High hairline & + & + & + & + & \\
\hline Downslanting palpebral fissures & + & + & + & & \\
\hline Prognathism & + & & & & \\
\hline Pointed chin & + & + & & & \\
\hline Large feet and hands & + & NR & NR & & \\
\hline Advanced bone age & + & + & + & NR & \\
\hline Heart defect & + & + & + & & \\
\hline Psycomotor delay & + & + & + & + & \\
\hline Speech delay & + & NR & NR & + & \\
\hline Intellectual disability & + & + & + & + & \\
\hline Behavioral problems & + & NR & NR & + & \\
\hline Seizures & + & + & + & & \\
\hline \multicolumn{6}{|l|}{ BWS clinical features } \\
\hline Macroglossia & & & & & + \\
\hline Earlobe creases & & & & & + \\
\hline Abdominal wall defect & & & & & + \\
\hline Neonatal hypogycemia & & & & & + \\
\hline Gene/Locus & NSD1 (5q35) & $\begin{array}{l}\text { 11p15 paternal } \\
\text { isodisomy }\end{array}$ & $\begin{array}{l}\text { KvDMR1 (11p15) } \\
\text { demethylation }\end{array}$ & $\begin{array}{l}\text { KvDMR1 (11p15) } \\
\text { demethylation }\end{array}$ & KvDMR1 (11p15) \\
\hline Phenotype & & Sotos syndrome & Sotos syndrome & Sotos-like & \\
\hline
\end{tabular}

Abbreviations: BWS, Beckwith-Wiedemann syndrome; NR, not reported.

$\mathrm{FD}$ and RD the code used to identify the patients in Baujat et al. ${ }^{3}$
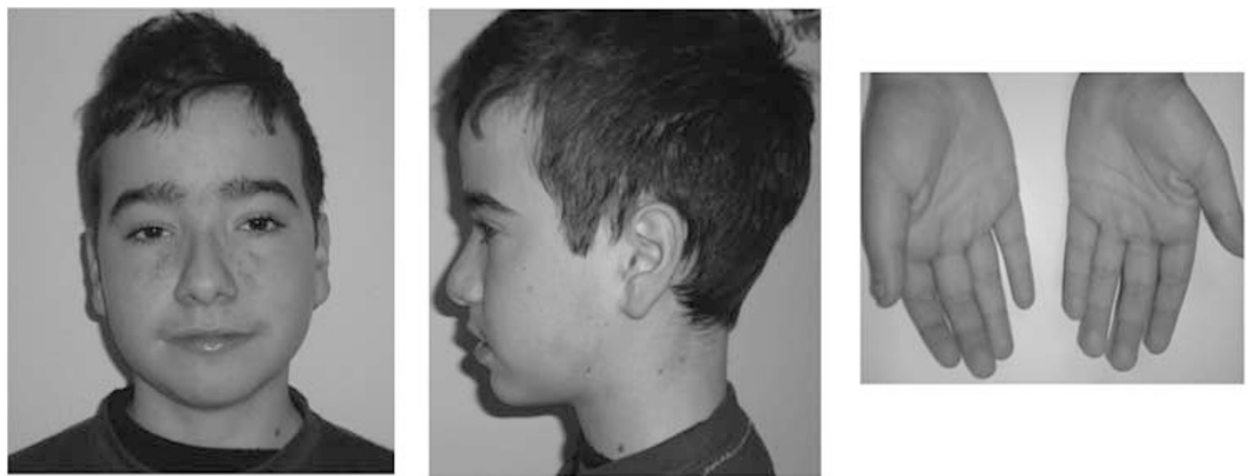

Figure 1 Hands, frontal and lateral facial views. All the images were taken when the patient was 12 years old with parental consent. 
a

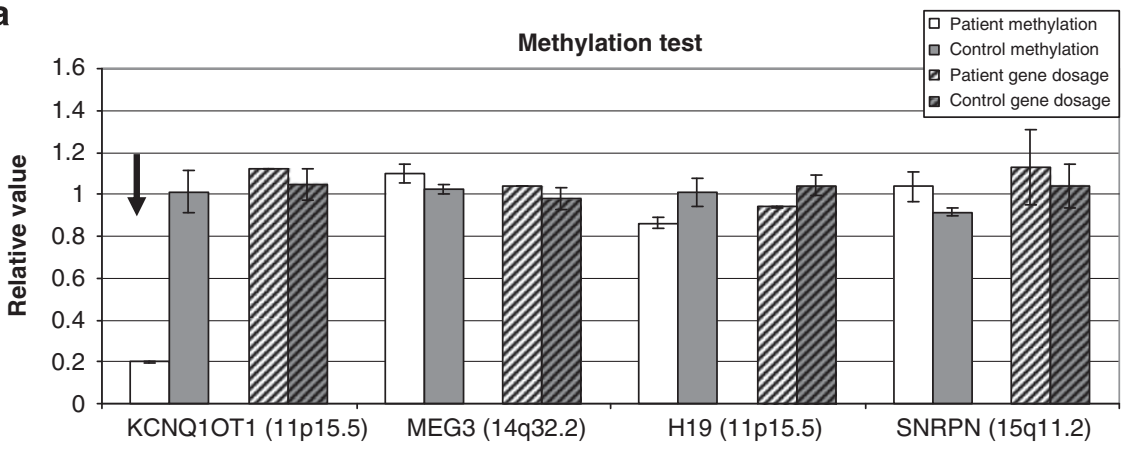

Imprintin region
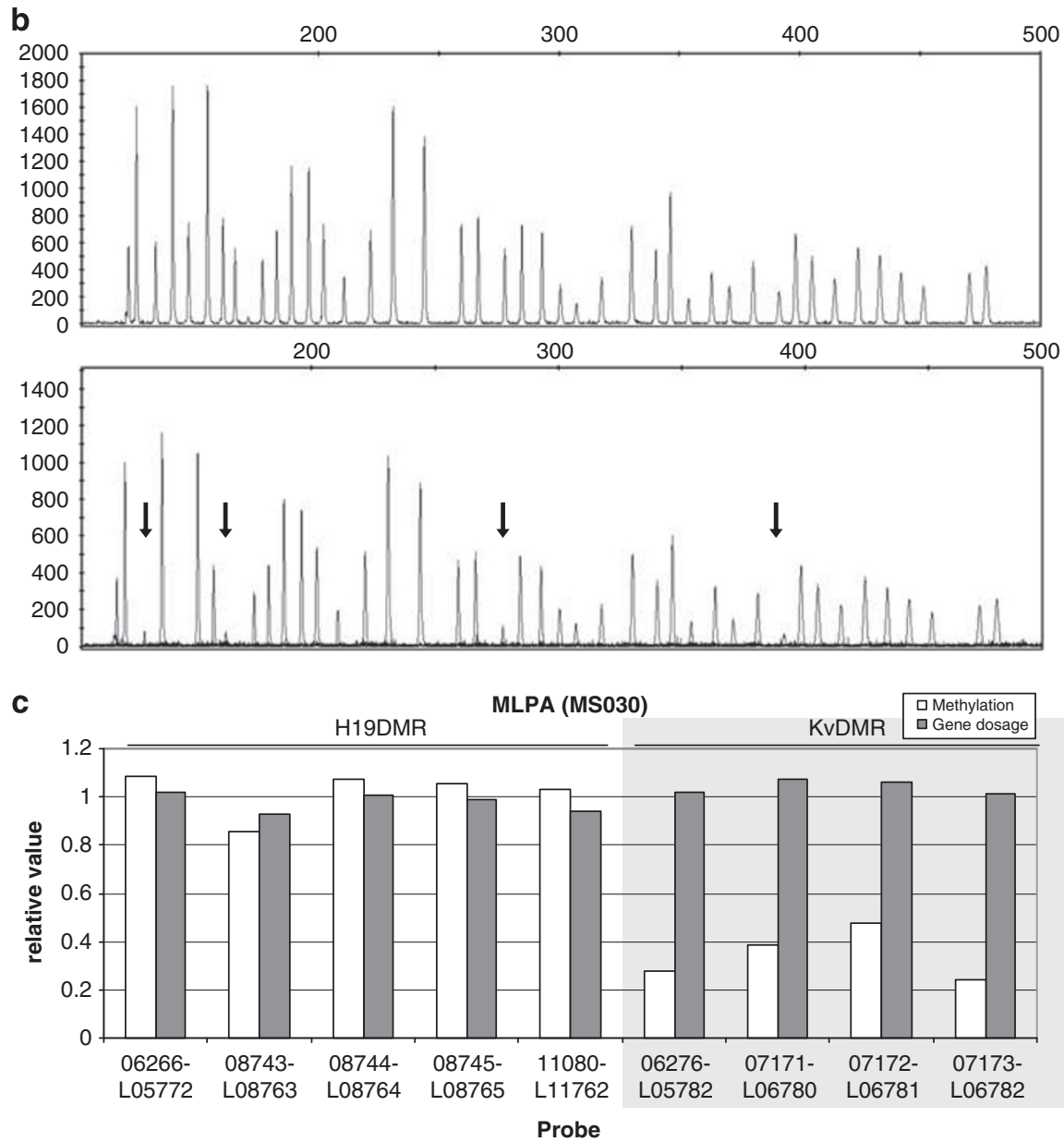

Figure 2 (a) Methylation studies. The dosage of four imprinting regions (KCNQ1OT1, MEG3, H19 and SNRPN) was measured in the patient and control samples simultaneously. It is considered as normal a relative value within $1 \pm 0.2$. The experiment was carried out twice by independent replicates. The hypomethylation of KCNQ1OT1 is indicated with a black arrow. (b and $\mathbf{c}$ ) MLPA studies. MLPA SALSA MSO30 patient results for the probes located in the two imprinting regions, H19DMR (H19) and KvDMR (KCNQ1OT1). Also, the normal relative values lie between 0.8 and 1.2 . (b) Methylation electropherogram from control sample (above) and from the patient sample (below). The KvDMR probes are indicated with black arrows. (c) Analyzed result of the MLPA. Raw data were normalized against five healthy controls. All the four CpG sites tested showed a loss of methylation in a similar degree to that seen in the semi-quantitative PCR amplification.

KCNQ1OT1 may well be used as a new diagnostic marker among Sotos-like patients of unknown cause. This marker would allow to establish a new (epi)genetically homogeneous entity and, in turn, it can facilitate the identification of the causing gene and/or a more accurate genetic counselling.

\section{CONFLICT OF INTEREST}

The authors declare no conflict of interest.

\section{ACKNOWLEDGEMENTS}

This study was supported by grant PI08/0648

(Fondo de Investigaciones Sanitarias, Ministerio de Ciencia e Innovación), FEDER (Fondo Europeo de DEsarrollo Regional), Fundación Ramón Areces and Fundación Eugenio Rodríguez Pascual. Sonia Mayo is supported by the Fundación para la Investigación del Hospital Universitario y Politécnico La Fe/Fundación Bancaja fellowship. Guiomar Perez de Naclares is partially funded by the I3SNS Program of the Spanish Ministry of Health (CP03/ 0064; SIVI 1395/09). Intza Garin is supported by FIS-program I3SNSCA10/01056 of the Spanish 
Ministry of Health. We are grateful for the collaboration of the patient and his family.

Sonia Mayo ${ }^{1,3}$, Intza Garin ${ }^{2,3}$, Sandra Monfort ${ }^{1}$, Mónica Roselló ${ }^{1}$, Carmen Orellana ${ }^{1}$, Silvestre Oltra ${ }^{1}$, Celia $\mathrm{Zazo}^{2}$, Guiomar Perez de Naclares ${ }^{2}$ and Francisco Martínez ${ }^{1}$

${ }^{1}$ Unidad de Genética y Diagnóstico Prenatal, Hospital Universitario y Politécnico La Fe, Valencia, Spain and ${ }^{2}$ Molecular Genetics Laboratory, Research Unit, Hospital Txagorritxu, C/José Achótegui, S/N, Vitoria-Gasteiz, Spain ${ }^{3}$ These authors contributed equally to this work and should be considered joint first authors. E-mail: francisco@gva.es
1 Tatton-Brown, K. \& Rahman, N. Sotos syndrome. Eur. J. Hum. Genet. 15, 264-271 (2007).

2 Baujat, G., Rio, M., Rossignol, S., Sanlaville, D., Lyonnet, S., Le Merrer, M. et al. Clinical and molecular overlap in overgrowth syndromes. Am. J. Med. Genet. C. Semin. Med. Genet. 137C, 4-11 (2005).

3 Baujat, G., Rio, M., Rossignol, S., Sanlaville, D., Lyonnet, S., Le Merrer, M. et al. Paradoxical NSD1 mutations in Beckwith-Wiedemann syndrome and $11 \mathrm{p} 15$ anomalies in Sotos syndrome. Am. J. Hum. Genet 74, 715-720 (2004).

4 Baujat, G \& Cormier-Daire, V Sotos syndrome. Orphanet. J. Rare Dis. 2, 36 (2007). Review.

5 Martínez, F., León, A. M., Monfort, S., Oltra, S., Roselló, M. \& Orellana, C. Robust, easy, and dosesensitive methylation test for the diagnosis of PraderWilli and Angelman syndromes. Genet. Test. 10, 174-177 (2006).

6 Monfort, S., Roselló, M., Orellana, C., Oltra, S., Blesa, D., Kok, K. et al. Detection of known and novel genomic rearrangements by array based comparative genomic hybridisation: deletion of ZNF533 and duplication of
CHARGE syndrome genes. J. Med. Genet. 45, 432-437 (2008)

7 Martínez, F., Monfort, S., Roselló, M., Oltra, S., Blesa, D., Quiroga, R. et al. Enrichment of ultraconserved elements among genomic imbalances causing mental delay and congenital anomalies. BMC Med. Genomics 3, 54 (2010).

8 Mackay, D. J., Callaway, J. L., Marks, S. M., White, H. E., Acerini, C. L., Boonen, S. E. et al. Hypomethylation of multiple imprinted loci in individuals with transient neonatal diabetes is associated with mutations in ZFP57. Nature Genet. 40, 949-951 (2008).

9 Meyer, E., Lim, D., Pasha, S., Tee, L. J., Rahman, F., Yates, J. R. et al. Germline mutation in NLRP2 (NALP2) in a familial imprinting disorder (BeckwithWiedemann Syndrome). PLoS Genet. 5, e1000423 (2009).

10 Murdoch, S., Djuric, U., Mazhar, B., Seoud, M., Khan, R., Kuick, R. et al. Mutations in NALP7 cause recurrent hydatidiform moles and reproductive wastage in humans. Nature Genet. 38, 300-302 (2006). 